\title{
Control of damping in perpendicularly magnetized thin films using spin-orbit torques
}

\author{
S. Saha $\odot,{ }^{1,2,{ }^{*}}$ P. Flauger, ${ }^{3, \dagger}$ C. Abert ${ }^{3}$ A. Hrabec,,${ }^{1,2,4}$ Z. Luo $\odot,{ }^{1,2}$ J. Zhou, ${ }^{1,2}$ V. Scagnoli, ${ }^{1,2}$ \\ D. Suess, ${ }^{3}$ and L. J. Heyderman ${ }^{1,2}$ \\ ${ }^{1}$ Laboratory for Mesoscopic Systems, Department of Materials, ETH Zurich, 8093 Zurich, Switzerland \\ ${ }^{2}$ Laboratory for Multiscale Materials Experiments, Paul Scherrer Institute, 5232 Villigen PSI, Switzerland \\ ${ }^{3}$ Christian Doppler Laboratory of Advanced Magnetic Sensing and Materials, Faculty of Physics, University of Vienna, 1090 Vienna, Austria \\ ${ }^{4}$ Laboratory for Magnetism and Interface Physics, Department of Materials, ETH Zurich, 8093 Zurich, Switzerland
}

(Received 19 March 2020; accepted 6 May 2020; published 1 June 2020)

\begin{abstract}
Magnetic damping plays a crucial role in the dynamics of magnetic systems. Hence, control over the damping is highly desirable for the development of magnetic devices. One of the possible ways to manipulate magnetic damping in a ferromagnetic material is the injection of spin currents generated by the spin Hall effect. The generated spin currents can be used to manipulate magnetic moments as well as the effective damping of the ferromagnetic material. In this paper, we demonstrate that the influence of the spin current on the damping is highly sensitive to the relative direction of the spin polarization and the precessional axis of the magnetization. The observations are important for various applications in magnetic storage devices and in microwave signal processing.
\end{abstract}

DOI: 10.1103/PhysRevB.101.224401

\section{INTRODUCTION}

Understanding and controlling the damping in ferromagnetic thin films is very important for emerging technologies including magnonics $[1,2]$ and spintronics [3,4]. While high-damping magnetic films with perpendicular magnetic anisotropy are required in data storage and memory devices to suppress the magnetization precession during writing, low damping is essential for spin-transfer torque magnetic random access memory to reduce the minimum required write current and for magnonic devices where long spin-wave propagation lengths are required to transfer information. For applications, tunability of the magnetic damping would be desirable. The damping can in general be irreversibly controlled by modifying the material with doping [5] or ion irradiation [6]. However, it has also been shown that the damping can be controlled reversibly by spin transfer torques $[7,8]$ that result from the injection of spin current. Here, the spin current interacts with the magnetization and modifies the damping $[9,10]$ in a ferromagnet, either by suppressing or by amplifying the magnetization precession [11]. The spin current can also be used to generate single-mode coherent auto-oscillation of a ferromagnetic film $[12,13]$.

A spin current can be generated via nonlocal spin injection [14], spin pumping [15], and the Rashba effect [16]. However, the most effective way to generate the spin current is via the spin Hall effect [17-19]. When a charge current flows through a heavy nonmagnetic metal layer such as $\mathrm{Pt}, \mathrm{Ta}$, and $\mathrm{W}$, electrons with opposite spins are deflected in opposite directions due to the spin-dependent scattering of the electrons, with local potentials created either by the heavy atoms or by defects

\footnotetext{
*susmitasaha.phy@gmail.com

†peter.flauger@univie.ac.at
}

(impurities). Finally, a transverse spin current is created due to the spin Hall effect [11].

In a ferromagnetic/heavy-metal bilayer film, the generated spin current in the heavy-metal layer is injected into the ferromagnetic layer and creates a torque acting on the magnetization. This is known as the spin-orbit torque (SOT) [20,21]. Under the influence of the SOT, the magnetization dynamics can be described by the Landau-Lifshitz-Gilbert equation with an additional SOT term $\boldsymbol{T}$ as

$$
\frac{\partial \boldsymbol{m}}{\partial t}=-\gamma \boldsymbol{m} \times \boldsymbol{H}^{\mathrm{eff}}+\alpha \boldsymbol{m} \times \frac{\partial \boldsymbol{m}}{\partial t}+\boldsymbol{T},
$$

where $\gamma$ is the gyromagnetic ratio, $\boldsymbol{m}$ is the magnetization vector, $\boldsymbol{H}^{\text {eff }}$ is the effective magnetic field, and $\alpha$ is the Gilbert damping parameter. The SOT term $\boldsymbol{T}$ can be decomposed into two torque terms, namely, the dampinglike torque $\left(\boldsymbol{T}_{\text {damp }}\right)$ and the fieldlike torque $\left(\boldsymbol{T}_{\text {field }}\right)$, expressed as

$$
\boldsymbol{T}_{\mathrm{damp}}=\eta_{\mathrm{damp}} \frac{j \gamma \hbar}{2 e \mu_{0} M_{s}} \boldsymbol{m} \times(\boldsymbol{m} \times \boldsymbol{p})
$$

and

$$
\boldsymbol{T}_{\text {field }}=\eta_{\text {field }} \frac{j \gamma \hbar}{2 e \mu_{0} M_{s}} \boldsymbol{m} \times \boldsymbol{p},
$$

where $M_{s}$ is the saturation magnetization, $j$ the current density, and $\boldsymbol{p}$ denotes the spin polarization. This polarization is perpendicular to both the direction of the charge current and the direction of the spin current following the right-hand rule: a charge current in the $x$ direction generates a spin current in the $z$ direction with a polarization in the $y$ direction. Both $\eta_{\text {damp }}$ and $\eta_{\text {field }}$ are dimensionless material-dependent quantities for the corresponding torques.

The dampinglike torque $\boldsymbol{T}_{\text {damp }}$ is the longitudinal component, which lies in the $(m, p)$ plane and acts like an effective damping. It has been demonstrated that $\boldsymbol{T}_{\text {damp }}$ could be strong 

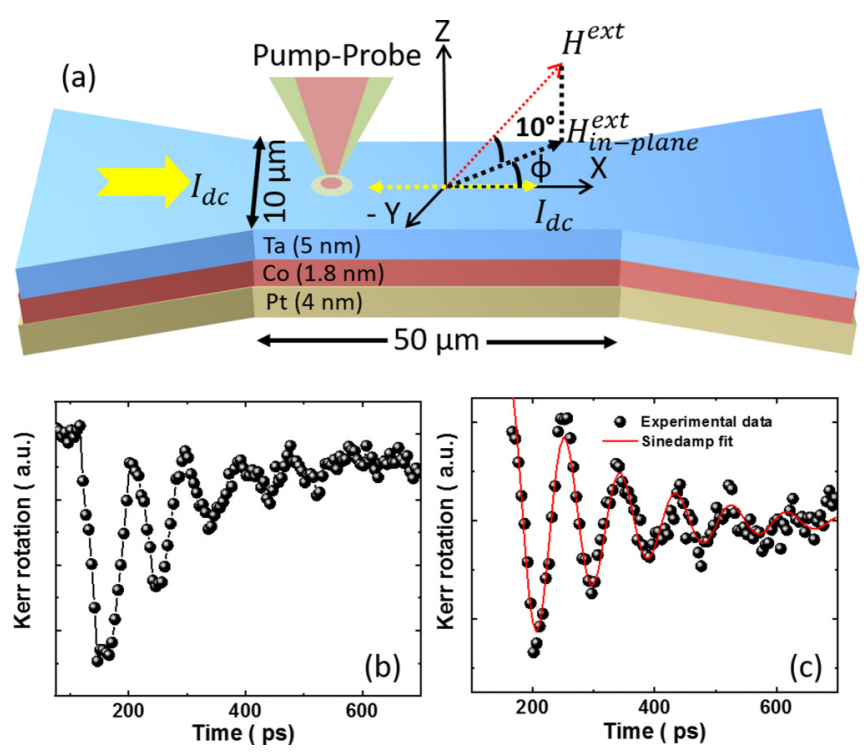

FIG. 1. (a) Schematic of the experimental setup in the presence of a DC current, $I_{\mathrm{dc}} . \phi$ is the angle between the effective external in-plane magnetic field $\boldsymbol{H}_{\text {in-plane }}^{\text {ext }}$ and the direction of the DC charge current $I_{\mathrm{dc}}$. (b) Typical time-resolved Kerr rotation data for the $[\mathrm{Pt} / \mathrm{Co} / \mathrm{Ta}]$ film at an applied magnetic field of $150 \mathrm{mT}$. (c) Timeresolved Kerr rotation data after a biexponential background subtraction of the data in (b). The solid red line corresponds to the fitting using the damped sine function given by Eq. (4).

enough to switch the magnetization of a ferromagnetic layer with both in-plane [22] and out-of-plane [23,24] anisotropy for sufficient current density. In contrast, the fieldlike torque,

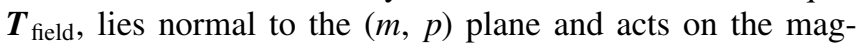
netization as an effective magnetic field. Thus, SOTs can influence the precessional dynamics [25] of a ferromagnetic thin film. In particular, they can also be used to tune the damping $(\alpha)$ of the magnetic material [26]. In general, the modification of the damping highly depends on the direction of the damping and fieldlike torques that derive from the spin polarization $\boldsymbol{p}$ [27]. However, an understanding of the exact role of the damping and fieldlike torques in the modulation of the damping is still needed.

In this paper, we demonstrate the influence of the relative angle between the precessional axis of the magnetization and the spin polarization on the damping $\alpha$ of a perpendicularly magnetized $[\mathrm{Ta}(3 \mathrm{~nm}) / \mathrm{Pt}(4 \mathrm{~nm}) / \mathrm{Co}(1.8 \mathrm{~nm}) / \mathrm{Ta}$ $(5 \mathrm{~nm}) / \mathrm{Pt}(1.5 \mathrm{~nm})]$ heterostructure using a combination of time-resolved magneto-optical Kerr-effect microscope (TRMOKE) experiments and micromagnetic simulations.

\section{EXPERIMENTAL OBSERVATIONS}

The film heterostructure was deposited using DC magnetron sputtering with a confocal sputter-up geometry at a base pressure of $10^{-8}$ Torr. The partial pressure of Ar used for deposition was 3 mTorr. For this film stack, the spin Hall angles for $\mathrm{Pt}$ and $\mathrm{Ta}$ are opposite to each other, which enhances the overall SOTs in the system. The film was patterned into electrodes using ion milling as shown in Fig. 1(a), with the length and width of the center stripe 50 and $10 \mu \mathrm{m}$, respectively. The damping of the ferromagnetic film [28] is measured in the time domain using TR-MOKE [29]. This setup is based on a two-color collinear pump-probe geometry. The Kerr rotation of the probe beam $(\lambda=1030 \mathrm{~nm}$ and pulse width $\approx 50 \mathrm{fs}$ ) is measured after exciting the sample with another laser beam of $\lambda=515 \mathrm{~nm}$ and a pulse width of $\approx 50 \mathrm{fs}$. Both the pump and the probe beams are focused on the sample using a microscope objective with numerical aperture N.A. $=0.65$. A $150 \mathrm{mT}$ external magnetic field $\left(\boldsymbol{H}^{\text {ext }}\right)$ is applied at a small angle $\left(10^{\circ}\right)$ to the sample plane. The pump pulse rapidly modifies the out-of-plane demagnetizing field and thereby induces precessional magnetization dynamics. The precessional dynamics appears as an oscillatory signal on top of the decaying time-resolved Kerr rotation as shown in Fig. 1(b). After subtraction of the biexponential background, the data are fitted using the following damped sine function $[7,30]$ :

$$
M(t)=M(0) \exp (-t / \tau) \sin (\omega t-\theta) .
$$

The fit is shown in Fig. 1(c). From the fit we estimate the damping $\alpha$ using the expression

$$
\alpha=1 / 2 \pi f \tau
$$

where $f$ is the precessional frequency, $\tau$ is the relaxation time corresponding to magnetization oscillation, and $\theta$ is the initial phase of oscillation. A fast Fourier transform of the background-subtracted data is performed to obtain the spin-wave spectra of the sample. In our experiment, the magnetization dynamics of the sample is measured upon the application of a DC current, $I_{\mathrm{dc}}$, along the $x$ direction [see Fig. 1(a)]. In the $x y$ plane, the angle $\phi$ between the effective external in-plane magnetic field $\boldsymbol{H}_{\text {in-plane }}^{\text {ext }}$ and the direction of the DC charge current $I_{\mathrm{dc}}$ is varied. The value of the damping parameter is recorded as a function of the DC current density for three values of $\phi$ as shown in Figs. 2(a)-2(c). The damping of the sample in the absence of a DC current is found to be $\alpha=0.087 \pm 0.008$ for $\phi=0^{\circ}, 45^{\circ}$, and $90^{\circ}$. The value of damping changes with the current density and has a strong dependence on $\phi$. There is a nonlinear variation in the damping for $\phi=0^{\circ}$ [see Fig. 2(a)], whereas for $\phi=45^{\circ}$ and $90^{\circ}$ the damping increases linearly with the applied current density [see Figs. 2(b) and 2(c)].

\section{MICROMAGNETIC SIMULATION}

In order to elucidate the experimental observations, the modified Landau-Lifshitz-Gilbert equation, Eq. (1), is solved using a macrospin approach with the micromagnetic simulation library magnum.fe [31]. The dynamics of the system is simulated where the system is excited by tilting the magnetization $\boldsymbol{m}$ a few degrees out of the equilibrium direction. The experimental system is modeled employing the magnetic parameters $M_{s}=9.35 \times 10^{5} \mathrm{~A} / \mathrm{m}, \alpha=0.082$, and $K_{\mathrm{u}}=$ $1.61 \times 10^{5} \mathrm{~J} / \mathrm{m}^{3}$, with $K_{\mathrm{u}}$ being the anisotropy constant for a uniaxial out-of-plane anisotropy. $M_{\mathrm{s}}$ and $K_{\mathrm{u}}$ are determined experimentally from in- and out-of-plane hysteresis loops of the sample using a superconducting quantum interference device with vibrating sample magnetometer (SQUID-VSM). In order to simulate the spin-orbit torque due to a DC current in the $x$ direction, we furthermore set the spin polarization $\boldsymbol{p}$ 

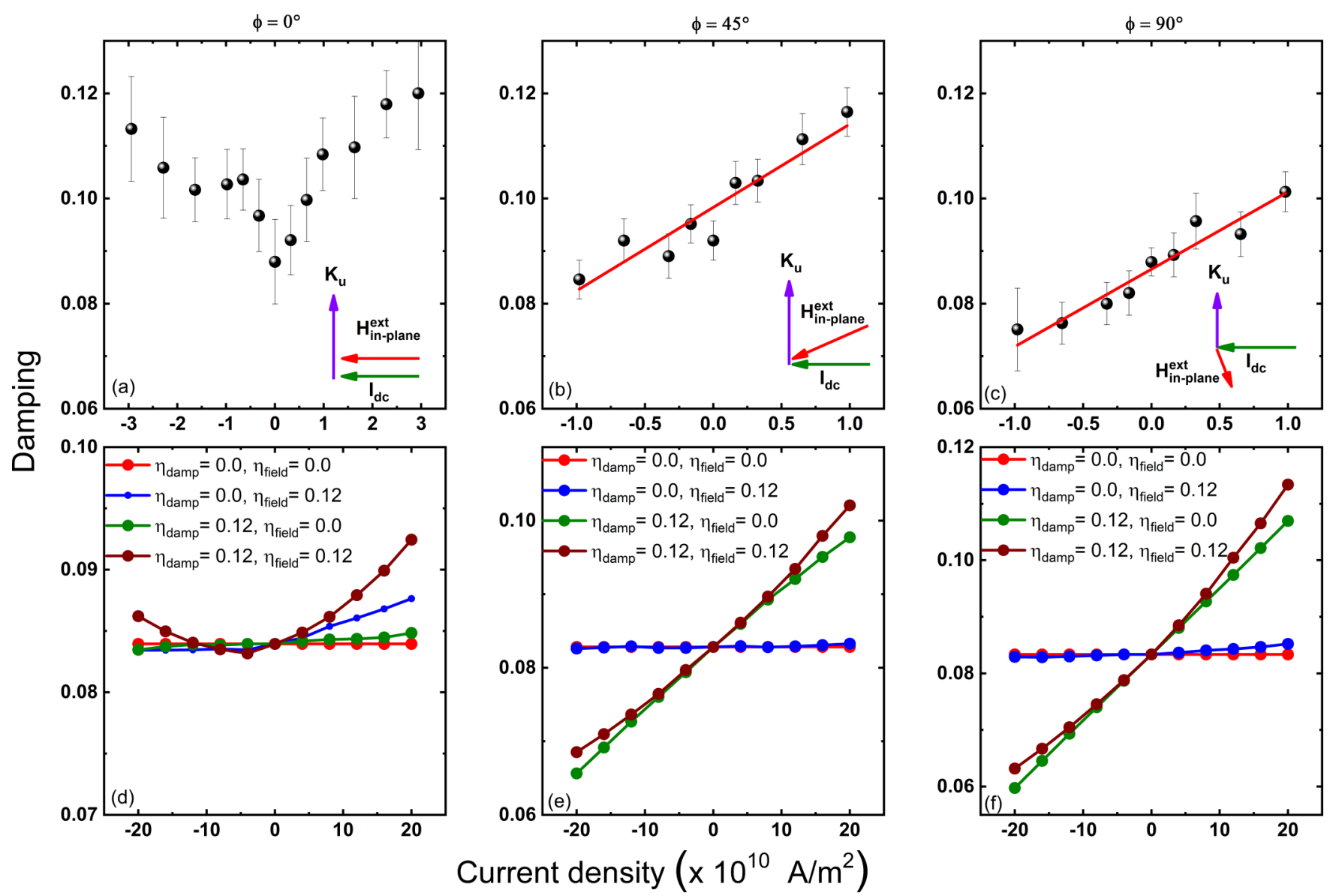

FIG. 2. Dependence of the damping parameter on the current density, determined from (a-c) TR-MOKE and (d-f) simulations for $\phi=0^{\circ}$, $45^{\circ}$, and $90^{\circ}$. Insets in (a)-(c): Schematics of the uniaxial out-of-plane anisotropy $\left(\mathrm{K}_{\mathrm{u}}\right)$, the current direction $\left(I_{\mathrm{dc}}\right)$ and in-plane component of the external magnetic field $\left(H_{\mathrm{in}-\mathrm{plane}}^{\mathrm{ex}}\right)$. In (d-f) dependence of the damping parameter on the current density for different combinations of $\eta_{\text {damp }}$ and $\eta_{\text {field }}$ is shown.

in the $y$ direction. The external field $\boldsymbol{H}^{\text {ext }}$ was set according to the experiment. The efficiency of the dampinglike torque $\eta_{\text {damp }}=0.12$ was taken from Refs. [32-34].

Figures 2(d)-2(f) show the dependence of the damping on the current density for different angles $\phi$, extracted from simulations with or without inclusion of the damping ( $\left.\eta_{\text {damp }}\right)$ and fieldlike ( $\left.\eta_{\text {field }}\right)$ torques. The plots indicate that the modification of damping is mainly due to the dampinglike torque, since the experiment and simulation show a good qualitative agreement in the presence of dampinglike torque. Possible reasons for the quantitative discrepancy include the sample roughness and heating by the laser, which would both enhance the magnitude of the damping in the experiment. For the nonlinear behavior as shown in Figs. 2(a) and 2(d), different excitations used in experiment and simulation can also lead to further disagreement. Additionally, in the experiment, the current density is calculated assuming a uniform distribution of the current through the entire stack whereas, in the simulation, the current density is estimated assuming that the current only flows in the heavy-metal layer. Nevertheless, both experiment and simulation exhibit a clear linear increase in the damping for field angles at $\phi=45^{\circ}$ and $90^{\circ}$, while the damping modification for $0^{\circ}$ exhibits a nonlinear trend with current density.
In order to modify the damping, the average torque over one precession of the magnetization has to exhibit a net component that points either towards or away from the precessional axis. As the precessional axis of magnetization is determined by the direction of the effective field, for a reasonable value of $j$, the modification of damping of the system is only possible, if $\eta_{\text {damp }} \neq 0$ and if the effective field has a component aligned with the spin polarization $\boldsymbol{p}$. To clarify this, we have determined the magnetization dynamics in a simplified system with a uniaxial anisotropy only. Here the magnetization dynamics is determined in the absence of an external magnetic field, while a current is applied along the $x$ direction. As a result, the uniaxial anisotropy field of the system is the only contribution to $\boldsymbol{H}^{\text {eff }}$. For the case of a fixed value of $\eta_{\text {damp }} \cdot j$, the orientation of the anisotropy axis $\boldsymbol{K}_{\mathrm{u}}$ regulates how much the damping will be modified by the dampinglike torque. As mentioned before, the spin polarization $\boldsymbol{p}$ is set along the $\hat{\boldsymbol{y}}$ direction. If $\boldsymbol{K}_{\mathrm{u}}$ and $\boldsymbol{p}$ are collinear $\left(\boldsymbol{K}_{\mathrm{u}} \| \hat{\boldsymbol{y}}\right)$, the precessional axis $\hat{\boldsymbol{r}}$ of the magnetization will be oriented along $\hat{\boldsymbol{y}}$ and therefore the dampinglike torque, which includes the term $\boldsymbol{m} \times(\boldsymbol{m} \times \boldsymbol{p})$, will point either towards or away from $\hat{\boldsymbol{r}}$, depending on the relative orientation of $\boldsymbol{m}$ and $\boldsymbol{p}$. Since the anisotropy is unidirectional, the magnetization may precess either in the half-plane pointing in the $+\hat{\boldsymbol{y}}$ direction 

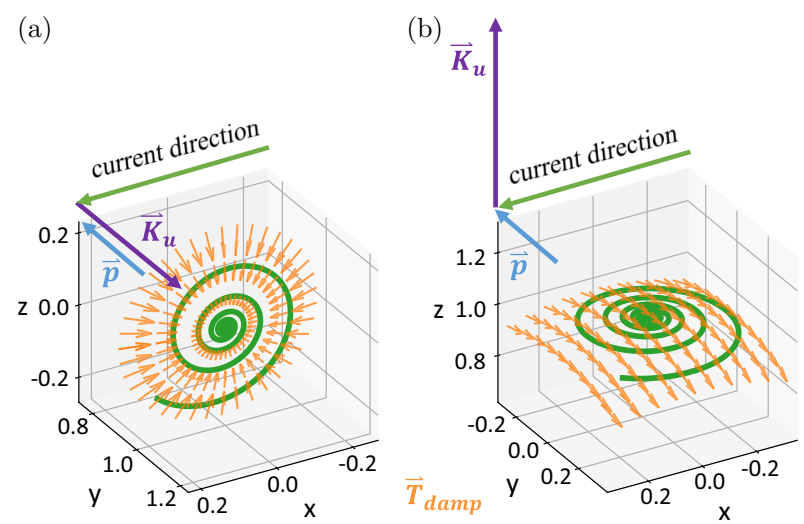

(c)

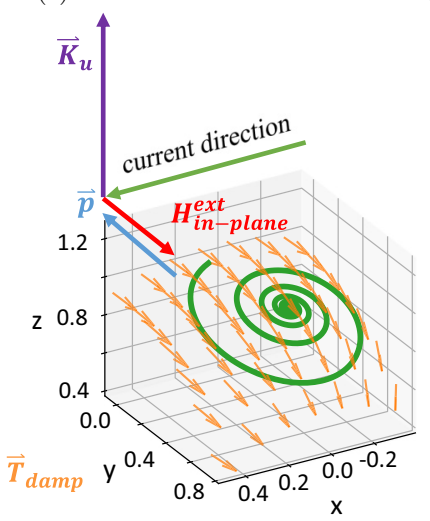

(d)

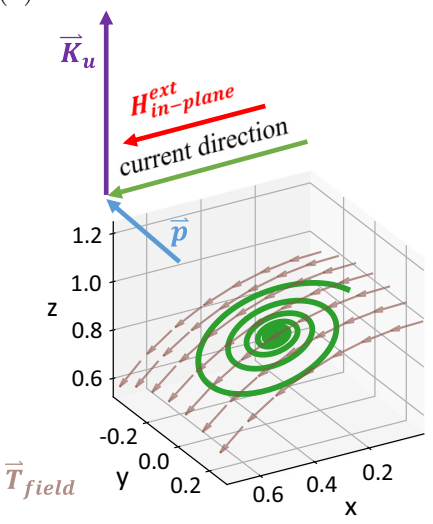

FIG. 3. Magnetization trajectory and spin-torque contribution for various model systems. The green line depicts the magnetization trajectory in three-dimensional space and the orange/brown arrows depict the spin-torque contributions acting on the magnetization. (a) A simplified system featuring only uniaxial anisotropy with $\boldsymbol{K}_{\mathrm{u}} \| \hat{y}$ and the dampinglike torque, and no external field. Since the current flows in the $\hat{x}$ direction, the spins injected into the system are polarized along $-\hat{y}$, i.e., $\boldsymbol{p} \| \hat{y}$. A damping modification is obtained since the torque (orange arrows) directs $\partial_{t} \boldsymbol{m}$ towards the precessional axis $\boldsymbol{r} \| \hat{y}$. (b) If the same system has an out-of-plane anisotropy, the damping of the system is not modified. (c) The experimental system with the in-plane component of $\boldsymbol{H}^{\text {eff }}$ parallel to $\hat{y}$. The dampinglike torque features a nonvanishing $x$ component directed towards the center of the rotation, thus increasing the damping. (d) The experimental system with only the fieldlike torque (brown arrows) and with the in-plane component of $\boldsymbol{H}^{\text {eff }}$ directed along $\hat{x}$. No damping modification arises due to the $\boldsymbol{m} \times \boldsymbol{p}$ symmetry of the fieldlike torque.

or in the half-plane pointing in the $-\hat{\boldsymbol{y}}$ direction. In Fig. 3(a), the first case where $\boldsymbol{m}$ is more or less pointing in the direction of $+\hat{\boldsymbol{y}}$ is depicted and a modification of damping is observed (i.e., the torque drives $\boldsymbol{m}$ towards $\hat{\boldsymbol{r}}$ ). In the second case, the torque will drive $\boldsymbol{m}$ away from $\hat{\boldsymbol{r}}$. However, if $\boldsymbol{K}_{\mathrm{u}}$ is oriented along $\hat{z}$ as shown in Fig. 3(b), there is no significant net torque $\boldsymbol{T}$ pointing towards or away from $\boldsymbol{r}$. Instead, a constant push or pull of the magnetization in a single direction on the unit sphere's surface will be observed.

The same explanation also holds true for the experimental system with an out-of-plane anisotropy $\left(\boldsymbol{K}_{\mathrm{u}} \| \hat{\boldsymbol{z}}\right)$ and in the presence of an external magnetic field which is slightly tilted out of the $x z$ plane. When $\boldsymbol{H}^{\text {eff }}$ has no $y$ component, there is no modification of the damping. However, if the $y$ component does not vanish, the precessional axis of the magnetization will be tilted away from the $x z$ plane and the dampinglike torque will develop an $x$ component that always points either towards or away from some convergence value of $m_{x}$. Thus, the damping will be modified. The extreme case of $\phi=90^{\circ}$ is depicted in Fig. 3(c). In contrast to the dampinglike torque, the fieldlike torque does not influence the damping as illustrated in Fig. 3(d), since $\boldsymbol{m} \times \boldsymbol{p}$, unlike $\boldsymbol{m} \times(\boldsymbol{m} \times \boldsymbol{p})$, is obtained from a conservative field.

\section{SUMMARY}

In summary, we have elucidated the role of the damping and fieldlike torques in the modification of damping of a perpendicularly magnetized thin film. In particular, it is mainly the dampinglike torque that is responsible for the observed modification. Indeed, the contribution of dampinglike torque greatly depends on the relative angle $\phi$ between the precessional axis of the magnetization and the spin polarization. At $\phi=45^{\circ}$ and $90^{\circ}$, the effective field has a component aligned with the spin polarization. As a result, the dampinglike torque points either towards or away from the precessional axis and a linear variation of damping with respect to the current density is observed. However, for $\phi=0^{\circ}$, the influence of the dampinglike torque on the damping approximately averages out and no damping modification is observed. Micromagnetic simulations not only show a good qualitative agreement with the experimental observations but also have demonstrated the contribution from the two different types of torque for different geometries.

In conclusion, we have shown that the damping in a magnetic film can be controlled by varying the angle between the precessional axis of magnetization and the spin polarization. These observations can further be extended to develop a low-power magnonic device, where spin-waves with different propagation lengths are required to transfer information.

The data that support this study are available via the Zenodo repository [35].

\section{ACKNOWLEDGMENTS}

S.S. acknowledges an ETH Zurich Post-Doctoral fellowship and the Marie Curie actions for People COFUND program. We acknowledge T. P. Dao and Prof. Pietro Gambardella, ETH Zurich, and Aurelien C. Manchon, associate professor at KAUST, for their valuable scientific inputs. J.Z. and L.J.H. acknowledge funding by the Swiss National Science Foundation (Project No. 200020 172774). P.F. and C.A. gratefully acknowledge financial support from the Austrian Federal Ministry for Digital and Economic Affairs and the National Foundation for Research, Technology and Development. A.H. was funded by the European Union's Horizon 2020 research and innovation program under Marie Sklodowska-Curie Grant Agreement No. 794207 (ASIQS). 
[1] S. Neusser and D. Grundler, Adv. Mater. 21, 2927 (2009).

[2] A. V. Chumak, V. I. Vasyuchka, A. A. Serga, and B. Hillebrands, Nat. Phys. 11, 453 (2015).

[3] A. Hoffmann and S. D. Bader, Phys. Rev. Appl. 4, 047001 (2015).

[4] D. Houssameddine, U. Ebels, B. Delaët, B. Rodmacq, I. Firastrau, F. Ponthenier, M. Brunet, C. Thirion, J. P. Michel, L. Prejbeanu-Buda, M. C. Cyrille, O. Redon, and B. Dieny, Nat. Mater. 6, 447 (2007).

[5] G. Woltersdorf, M. Kiessling, G. Meyer, J.-U. Thiele, and C. H. Back, Phys. Rev. Lett. 102, 257602 (2009).

[6] A. Ganguly, S. Azzawi, S. Saha, J. A. King, R. M. RowanRobinson, A. T. Hindmarch, J. Sinha, D. Atkinson, and A. Barman, Sci. Rep. 5, 17596 (2015).

[7] S. Mondal, S. Choudhury, N. Jha, A. Ganguly, J. Sinha, and A. Barman, Phys. Rev. B 96, 054414 (2017).

[8] A. Capua, T. Wang, S.-H. Yang, C. Rettner, T. Phung, and S. S. P. Parkin, Phys. Rev. B 95, 064401 (2017).

[9] K. Ando, S. Takahashi, K. Harii, K. Sasage, J. Ieda, S. Maekawa, and E. Saitoh, Phys. Rev. Lett. 101, 036601 (2008).

[10] V. E. Demidov, S. Urazhdin, E. R. J. Edwards, and S. O. Demokritov, Appl. Phys. Lett. 99, 172501 (2011).

[11] L. Liu, T. Moriyama, D. C. Ralph, and R. A. Buhrman, Phys. Rev. Lett. 106, 036601 (2011).

[12] V. E. Demidov, S. Urazhdin, H. Ulrichs, V. Tiberkevich, A. Slavin, D. Baither, G. Schmitz, and S. O. Demokritov, Nat. Mater. 11, 1028 (2012).

[13] M. Evelt, C. Safranski, M. Aldosary, V. E. Demidov, I. Barsukov, A. P. Nosov, A. B. Rinkevich, K. Sobotkiewich, X. Li, J. Shi, I. N. Krivorotov, and S. O. Demokritov, Sci. Rep. 8, 1269 (2018).

[14] Y. Fukuma, L. Wang, H. Idzuchi, S. Takahashi, S. Maekawa, and Y. Otani, Nat. Mater. 10, 527 (2011).

[15] H. J. Jiao and G. E. W. Bauer, Phys. Rev. Lett. 110, 217602 (2013).

[16] I. M. Miron, G. Gaudin, S. Auffret, B. Rodmacq, A. Schuhl, S. Pizzini, J. Vogel, and P. Gambardella, Nat. Mater. 9, 230 (2010).

[17] J. E. Hirsch, Phys. Rev. Lett. 83, 1834 (1999).

[18] Y. Niimi and Y. Otani, Rep. Prog. Phys. 78, 124501 (2015).

[19] V. E. Demidov, S. Urazhdin, E. R. J. Edwards, M. D. Stiles, R. D. McMichael, and S. O. Demokritov, Phys. Rev. Lett. 107, 107204 (2011).

[20] A. Manchon, J. Železný, I. M. Miron, T. Jungwirth, J. Sinova, A. Thiaville, K. Garello, and P. Gambardella, Rev. Mod. Phys. 91, 035004 (2019).
[21] M. Baumgartner, K. Garello, J. Mendil, C. O. Avci, E. Grimaldi, C. Murer, J. Feng, M. Gabureac, C. Stamm, Y. Acremann, S. Finizio, S. Wintz, J. Raabe, and P. Gambardella, Nat. Nanotechnol. 12, 980 (2017).

[22] L. Liu, C.-F. Pai, Y. Li, H. W. Tseng, D. C. Ralph, and R. A. Buhrman, Science 336, 555 (2012).

[23] L. Liu, O. J. Lee, T. J. Gudmundsen, D. C. Ralph, and R. A. Buhrman, Phys. Rev. Lett. 109, 096602 (2012).

[24] A. van den Brink, G. Vermijs, A. Solignac, J. Koo, J. T. Kohlhepp, H. J. M. Swagten, and B. Koopmans, Nat. Commun. 7, 10854 (2016).

[25] M. Evelt, V. E. Demidov, V. Bessonov, S. O. Demokritov, J. L. Prieto, M. Muñoz, J. Ben Youssef, V. V. Naletov, G. de Loubens, O. Klein, M. Collet, K. Garcia-Hernandez, P. Bortolotti, V. Cros, and A. Anane, Appl. Phys. Lett. 108, 172406 (2016).

[26] A. Hamadeh, O. d'Allivy Kelly, C. Hahn, H. Meley, R. Bernard, A. H. Molpeceres, V. V. Naletov, M. Viret, A. Anane, V. Cros, S. O. Demokritov, J. L. Prieto, M. Muñoz, G. de Loubens, and O. Klein, Phys. Rev. Lett. 113, 197203 (2014).

[27] S.-h. C. Baek, V. P. Amin, Y.-W. Oh, G. Go, S.-J. Lee, G.-H. Lee, K.-J. Kim, M. D. Stiles, B.-G. Park, and K.-J. Lee, Nat. Mater. 17, 509 (2018).

[28] J. Walowski, M. D. Kaufmann, B. Lenk, C. Hamann, J. McCord, and M. Munzenberg, J. Phys. D: Appl. Phys. 41, 164016 (2008).

[29] S. Saha, R. Mandal, S. Barman, D. Kumar, B. Rana, Y. Fukuma, S. Sugimoto, Y. Otani, and A. Barman, Adv. Funct. Mater. 23, 2378 (2013).

[30] S. Pal, B. Rana, O. Hellwig, T. Thomson, and A. Barman, Appl. Phys. Lett. 98, 082501 (2011).

[31] C. Abert, L. Exl, F. Bruckner, A. Drews, and D. Suess, J. Magn. Magn. Mater. 345, 29 (2013).

[32] M.-H. Nguyen, D. C. Ralph, and R. A. Buhrman, Phys. Rev. Lett. 116, 126601 (2016).

[33] A. Giordano, M. Carpentieri, A. Laudani, G. Gubbiotti, B. Azzerboni, and G. Finocchio, Appl. Phys. Lett. 105, 042412 (2014).

[34] L. Zhu, K. Sobotkiewich, X. Ma, X. Li, D. C. Ralph, and R. A. Buhrman, Adv. Funct. Mater. 29, 1805822 (2019).

[35] S. Saha and P. Flauger, Control of damping in perpendicularly magnetized thin films using spin-orbit torques (Version 1.0), Zenodo, doi: 10.5281/zenodo.3837274. 